\title{
TECHNICAL SPECIFICATION FOR THE VALIDATION OF REMOTE SENSING PRODUCTS
}

\author{
Y. Ge ${ }^{\text {a }}$ *, X. Li ${ }^{\text {b }}$, M. G. Hu ${ }^{\text {a }}$ J.H. Wang ${ }^{\text {a }}$, R. Jin ${ }^{\text {b }}$, J. F. Wang ${ }^{\text {a }}$, R.H. Zhang ${ }^{\text {a }}$ \\ ${ }^{a}$ Institute of Geographic Sciences and Natural Resources Research, Chinese Academy of Sciences, 11A, Datun Road, \\ Beijing, China \\ ${ }^{\mathrm{b}}$ Cold and Arid Regions Environmental and Engineering Research Institute, Chinese Academy of Sciences, 320 \\ Donggang West Road, Lanzhou, China
}

KEY WORDS: Technical Specification, Validation, Remote Sensing Product, Heterogeneity

\begin{abstract}
:
The validation of remote sensing products (RSPs) is fundamental work before the proper use of RSPs. This paper mainly introduces the technical specification for the validation of remote sensing product, which is used to guide user how to validate the available products. The validation of remote sensing products (VRSP) mainly there are two levels. The first level focuses on the validation at pixel scale and the other is the product level which contains a number of pixels that are distributed in different land use types which are selected to represent both the space-time characteristics of surface parameters investigated. At pixel scale, the validation procedure mainly consists of three steps: sampling design for ground observation, data collection and estimation the mean value at pixel scale. The data types of ground observation include the wireless sensor network, which are used to capture multi-scale heterogeneities of surface parameters, and the footprint which could be a flux observation such as eddy covariance (EC) and large aperture scintillometer (LAS). After finishing the validation at pixel scale, the second-level validation for the whole remote sensing product is followed. The assessment with indexes is then used to evaluate the performance of the validation. Last, a validation report on the description of the validation will be included in the technical specification.
\end{abstract}

\section{INTRODUCTION}

Currently, remote sensing products (RSPs) have been widely applied to several fields of geosciences including atmospheric science, geology, geography, ecology, environmental science and soil science. With rich kinds of remote sensing products, it is gradually recognized that the validation of RSP should be implemented prior to the use of RSPs as it has been found that some products might overestimate/underestimate the accuracy of target variable (Li et al., 2008 and 2009; Sun et al., 2009; Zhang, 2009; Liu et al., 2010; Zhang et al., 2010; Jia et al., 2012). The validation of remote sensing products (VRSP) is a process which independently assesses the accuracy and precision of RSP with ground observation data (Li et al, 2009 and Li et al., 2012). This paper mainly introduces the technical specification for the validation of remote sensing product, which is used to guide user how to validate the available products. In the VRSP, there are two levels of validations. The first level focuses on the validation at pixel scale which involves the sampling design for ground observation data and the statistical inference from samples to estimate the value at pixel scale. The other is the product level which contains a number of pixels that are distributed in different land use types which are selected to represent both the space-time characteristics of surface parameters investigated. The ground observation data types of ground observation include the wireless sensor network, which are used to capture multi-scale heterogeneities of surface parameters, and the footprint which could be a flux observation such as eddy covariance (EC) and large aperture scintillometer (LAS). This technical specification not only includes the general situations for the VRSP such as sampling design for single variable at static condition but also includes the more complex situations such as sampling design for multiple variables static/dynamic conditions (Ge et al.,
2012). In particular, this specification provides validation strategies for RSP in homogeneous and heterogeneous surface conditions (Li 2006). Furthermore, some scaling techniques such as linear scaling conversion and non-linear scaling from samples to pixel scale are also involved in this specification.

\section{FRAMEWORK AND COMPONENTS OF TECHNICAL SPECIFICATION}

RS products validation contains a lot of technique and method details due to different characteristics of rich kinds of products (Cihlar et al., 1997; Justice et al., 2000). Here a general framework and its components are provided for RS products validation. In the framework, four components are defined and specified (Figure 1). The research scopes and related conception, notations, abbreviations and consistency are defined in the basic items part. Twenty six kinds of products are contained in the specification, involving atmospheric products (such as downward shortwave radiation, PAR and AOD), categorical land surface products (such as land cover type, surface freezing and thawing), continuous land surface products (such as NDVI, NPP and soil moisture), polar region categorical products (such as Antarctic coastline, thickness of ice sheet and grounding line), and high spatial resolution RS images. The validation is based on ground observation. Pixel scale estimation is first level validation of RS products. Then the whole spatial and temporal products are validated based on the first level pixel scale validation. Last, the validation results, including validation method and quality indicator, are included into a validation report for users. 


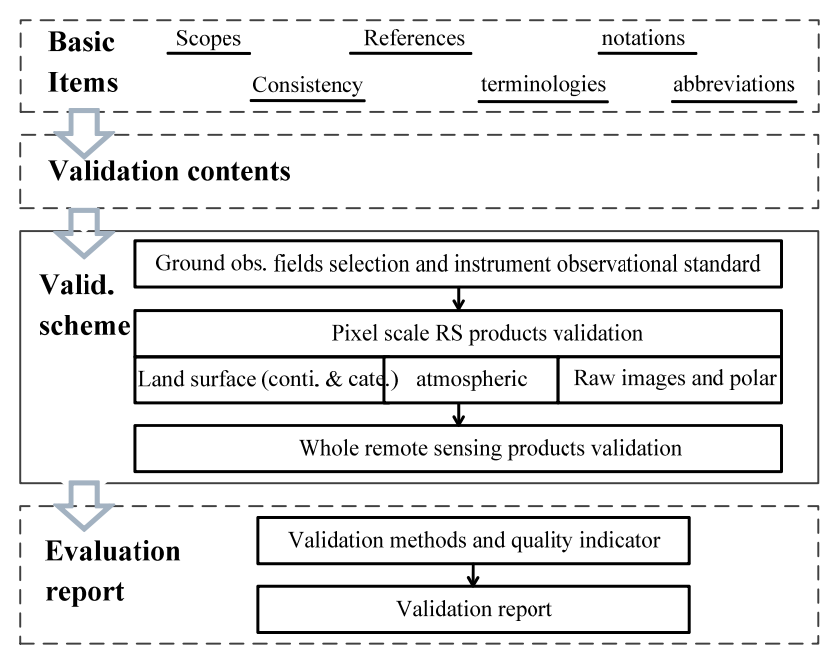

Figure 1. General framework of RSP technical specification

\subsection{Ground observation sites selection and instrument observational standard}

Ground observation sites (GOS) could be a place which has a relatively low standard deviation of the reflectance for vicarious remote sensing product validation. For a different purpose of validation, GOS can be divided into two categories: specialization GOS and integrated GOS. Specialization GOS is designed to validate the pixel which has a homogeneity land cover. For each type of land cover, a GOS is needed in which to observe the parameter values and validate the corresponding remote sensing product. Integrated GOS is designed to validate several types of remote sensing products. It is necessary to select and characterize a number of candidate GOS in terms of the criteria of spatial homogeneity, temporal stability at all scales, and cloud cover. The size of the selected site should be compatible with the size of pixels of product. And $2 \times 2$ to $5 \times 5$ pixels size are recommended, for the consideration of pixel shift. Furthermore, a number of calibrated instruments will be installed for VRSP. As the result of validation of RSP changes over time, the ground-based observation should be consistent with the satellite passed time.

\subsection{Pixel-scale validation of RS products}

Pixel is the basic composition of RS product. It is the first stage to validate whether the pixel value is in accord with the actual parameters. A common direct validation method for the pixelscale product is to compare the product with an independently observed data, which observes the same ground parameter at the same time and location (Li et al, 2009 and Li et al., 2012). The observed data can be obtained through a ground network designed by spatial sampling or foot printing. Apart from the direct validation, indirect validation is can also be done by comparison with the output from models that simulating the geographical process well. Both the theory of the model and the data entered into the model should be with high accuracy to reflect the actual ground parameter. Another method to validate the pixel products is cross validation with other same type RS products, whose accuracy is known and high.

Among the three validation methods, direct validation should be adopted during all kinds of RS products. Considering to significant difference in spatial sampling and aggregation method for direct validation, RS products can be classified into following three categories:

(1) Continuous products of land surface: the pixel value can be treated as a continuous variable whose value changes continuously. The method of aggregation from ground samples distributed in a selected pixel is the spatial sampling, and the aggregated value is a weighted sum of all samples where the sample weight is determined by the sampling method.

(2) Categorical products of land surface: the pixel value of this type product is a categorical variable. The value is usually limited in two or more categories. There is no order and size between the values. The pixel can also be validated by a ground based spatial sampling, while the aggregation method is different from the continuous products. The value whose area dominated the pixel region can be selected as the pixel value.

(3) Atmospheric products: different from the above land surface RS products, atmospheric products are little affected by the ground surface condition. A sample measurement in the pixel can usually represent the whole pixel.

\subsection{Whole RS product Validation}

Single pixel-scale validation of RS product evaluates the accuracy of selected pixels. But it is not enough to evaluate the accuracy of whole RS product, which might change across the space and time. To validate the whole RS product, the spacetime characteristics of ground parameter should be covered in the validation process (Figure 2). Pixel-scale validation is the base of the whole RS product validation. To make sure the product's accuracy is rather stable along with the time, it is necessary to observe and validate the selected pixels continuously. For spatial variation of RS products in a heterogeneous region, typical ground surfaces should be covered by spatial samples, where observation data are obtained simultaneously. The whole accuracy is then estimated by sampled pixels. Validation reports should be supplied to users to adjust whether the products meet their requirements.

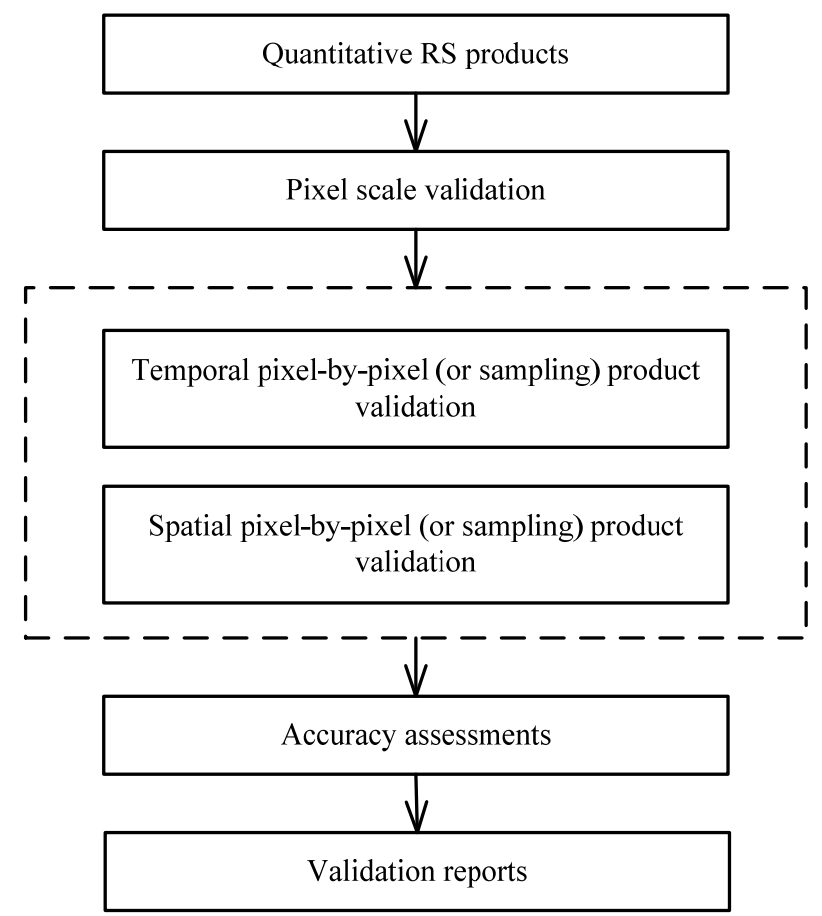

Figure 2. RS products validation

There is another particular type of RS products for Polar Regions, such as Antarctic coastline, thickness of ice sheet and grounding line. Validation of these products involves not only attributes but also geometrical features. The contents to be validated include data integrity, spatial/temporal resolutions, 
horizontal/vertical accuracy, and so on. Meanwhile, it is hard to be directly checked at the in-situ region. Thus it is often validated by indirect method, such as comparison with same type higher accurate products.

\subsection{High resolution satellite imagery quality validation}

High resolution satellite imagery quality validation is different from the quantitative remote sensing products. The following four indexes need to be evaluated in practice.

(1) Modulated transferring function (MTF). MTF is determined by a so-called sample image method and is commonly evaluated on remote sensing level 1 product.

(2) Radiation quality. Validation of radiation quality includes: image signal to noise ratio; dynamic range of radiation; relative accuracy of radiometric calibration; absolute accuracy of radiometric calibration.

(3) Geometric quality. Validation of geometric quality includes: calculation of bias matrix; confirmation of attitude data; validation of single image positioning accuracy, regional image positioning accuracy, internal distortion and registration accuracy.

(4) Ground pixel resolution. Ground pixel resolution is acquired by decomposing the difference between ground control points and the actual coordinates into course and lateral directions, then divide the decomposition by the number of pixels in course and lateral directions respectively.

\subsection{Assessment for the performance of VRSP}

The assessment system includes the following parts:

(1) Assessment at pixel scale. It means that only one or a number of pixels in remote sensing product are tested for a particular time period. In this circumstance, we can directly compare the remote sensing inversion value with the pixel "true" value to get the accuracy of product with the index of absolute error or relative error.

(2) Assessment in time domain. This assessment is to test the stability of the remote sensing product accuracy as time varies. In this condition, we can directly prove the accuracy of remote sensing product by testing the time series variation between the remote sensing product and pixel "true" value.

(3) Assessment in space domain. For the multiple pixels validation, several validation stations need to be established simultaneously to obtain the "true" value of multiple pixels for one remote sensing product. Meanwhile, the above multiple pixels "true" value needs to be compared with the remote sensing product, thus gaining the accuracy of whole product.

(4) The validation for a category remote sensing product. For this kind of remote sensing product, a number of calibration samples need to be established in each category to evaluate the accuracy of products by using the commonly used error matrix assessment method.
Table 1. Required information for different sampling models

\begin{tabular}{|c|c|c|}
\hline Classification & Validation index & Formula \\
\hline Pixel scale & $\begin{array}{l}\text { absolute error, } \\
\Delta \\
\text { relative error, } \delta\end{array}$ & $\begin{array}{l}\Delta=X-L \\
\delta=\Delta / L \times 100 \%\end{array}$ \\
\hline Time series & $\begin{array}{l}\text { time series } \\
\text { correlation, } r_{x y}\end{array}$ & $r_{x y}=\frac{\sum_{i=1}^{n}\left(x_{i}-\bar{x}\right)\left(y_{i}-\bar{y}\right)}{\sqrt{\sum_{i=1}^{n}\left(x_{i}-\bar{x}\right)^{2}} \sqrt{\sum_{i=1}^{n}\left(y_{i}-\bar{y}\right)^{2}}}$ \\
\hline Spatial domain & $\begin{array}{l}\text { mean absolute } \\
\text { difference, } \bar{\Delta} \\
\text { mean relative } \\
\text { error, } \bar{\delta}\end{array}$ & $\begin{array}{l}\bar{\Delta}=\frac{1}{n} \sum_{i=1}^{n}\left|\Delta_{i}\right| \\
\bar{\delta}=\frac{1}{n} \sum_{i=1}^{n} \delta_{i}\end{array}$ \\
\hline & RMSE & $\mathrm{RMSE}=\sqrt{\frac{1}{n} \sum_{i=1}^{n} \Delta_{i}^{2}}$ \\
\hline $\begin{array}{l}\text { Category } \\
\text { products }\end{array}$ & error matrix & $\begin{array}{l}\text { producer accuracy, user } \\
\text { accuracy; } \\
\text { overall accuracy, } \\
\text { and kappa coefficient }\end{array}$ \\
\hline
\end{tabular}

Notation: $X$ and $L$ are the measurement value and pixel "true" value. $n$ is the number of meansure, $x, y$ are the two time series measurement, and $\bar{x}, \bar{y}$ are the mean values of two time series.

\subsection{VRST Report}

A validation report will be issued after finishing the remote sensing product validation. This report includes the four following parts. (1) The main description of the target remote sensing product. It includes the name of validation product, data source and metadata, temporal and spatial resolution, spatial coverage, and retrieval algorithms et al. (2) The summary of validation method. (3) Uncertainty analysis during the process of validation. It is mainly about the analysis of error sources form the observation equipment error, model error. (4) The conclusion of the validation and the accuracy of the product. (5) People, units, time, location, and signature of the validation.

\section{METHODS FOR VRSP}

In the pixel-scale validation stage, sampling is the basic method to obtain the "true" value of the selected pixel. For a homogeneous pixel region, such as atmospheric products, a small number of samples are enough to capture most information in the region. While for a heterogeneous pixel region, more samples are usually needed to reach a high estimated accuracy. Moreover, the samples should be carefully designed to obtain an unbiased estimation. Thus, it is an important issue in the pixel-scale validation that how to select appropriate sampling method.

\subsection{Sampling method in pixel-scale $\mathrm{RS}$ product validation}

Sampling is an often used to understand objects in many disciplines. Compared to general survey, sampling has some particular advantages (Cochran, 1977; Haining, 2003). First, it is rapid and efficient. A sampling survey can be done in a short time, and it enables continuous observation possible to validate spatial/temporal variation of RS products. Second, it is fund saving. Only a small number of samples are surveyed. Third, a well designed sampling scheme can decrease systematic errors during the investigation process and increase the estimation accuracy. 
A suitable sampling method should be determined according to the particular characteristics in the selected pixel region. Spatial autocorrelation and homogeneity are two of the most important characteristics in sampling design. When there is no spatial autocorrelation of object ground attribute in the pixel region, classical sampling methods (such as simple random sampling, systematic sampling and stratified sampling) can be used in the product validation. Otherwise, spatial sampling methods are recommended (Table 2). Stratified sampling and MSN model take into consideration of stratification where it is necessary if the region is heterogeneous (Wang et al., 2009; Stein and Ettema, 2003). In Kriging and MSN based sampling, there is an object function to minimize variance of the estimated population (Hu and Wang, 2011; Ge et al., 2012). So the result is a best linear unbiased estimator of the real population. When considering the autocorrelation information in Kriging and MSN, semivariogram models should be built before the sampling process, which usually requires some prior information about the pixel region.

\begin{tabular}{|c|c|c|c|c|}
\hline $\begin{array}{c}\text { Sampling } \\
\text { Model }\end{array}$ & $\begin{array}{c}\text { Auto- } \\
\text { correlation }\end{array}$ & Stratum & $\begin{array}{l}\text { Object } \\
\text { function }\end{array}$ & Prior info. \\
\hline Simple random & $x$ & $x$ & $x$ & o \\
\hline Stratified & $x$ & $\sqrt{ }$ & $x$ & $\sqrt{ }$ \\
\hline Kriging & $\sqrt{ }$ & $x$ & $\sqrt{ }$ & $\sqrt{ }$ \\
\hline MSN & $\sqrt{ }$ & $\sqrt{ }$ & $\sqrt{ }$ & $\sqrt{ }$ \\
\hline
\end{tabular}

Table 2. Required information for different sampling models

\subsection{Statistical inference from samples in pixel-scale RS product validation}

Statistical inference is the next stage after samples is obtained under the determined sampling scheme. It estimates the pixel value from the samples. The estimation model can be the same as that used in sampling stage, including both classical sampling models and spatial sampling models. However, they need not to be the same when new information is found that another model is more suitable for the new situation. For example, in sampling stage, samples are collected by random sampling model while strong spatial autocorrelation is found from the collected samples. Then, Kriging model or MSN model rather than the simple random model can be selected to estimate the pixel value.

Bias sample is another problem in continuous observation. There would be systematic error between the results from bias sample and true value if the bias is not processed properly. Although the samples are well designed at the beginning of the research, attrition bias is often occurred due to samples missing or unavailable. When correlation between samples is not change and can be calculated from historical data, the bias could be corrected by posterior analysis, for example, the B-SHADE model (Wang et al., 2011).

Above statistical inference method is based on the assumption that the surface parameter can be estimated with a linear system. However, in practice, surface phenomena maybe disorganized and cannot be estimated with a linear method, but they still have a certain mathematical laws when analyzed by fractal theory, and it can be estimated with a fractal upscaling method (Kim and Berg 2000). Fractal dimension is used to quantitative describe this quality. Through the self-similarity dimension, fractal theory analyzes fractal dimension changes of different levels in nonlinear system, contacts pattern characteristics on different scales and prompts the similarities and differences of the multi-scale system characteristics, so as to provide the basis for upscaling.

\section{RESULTS AND DISCUSSION}

Validation is a fundamental work for the application of RS products. In this technical specification, a general framework and principles of VRSP are defined. Some specific method and techniques are particularly introduced for pixel scale and whole product validation, including the ground observation field selection criteria, instrument observation standards and methods, validating flow for three types of pixel scale RS production (continuous products of land surface, categorical products of land surface and atmospheric products), evaluation indicators, and sampling and inference methods. However, there are some problems need to be further investigated. One is sampling and inference for dynamic ground objects. When the object changes greatly due to some external factors, it is difficult to design a sole sample scheme to capture all dynamic information. An optional method is to design samples by simple random sampling or systematic sampling, and the inference model is data adaptive according to characteristic of the collected samples. Pixel validation by footprint is another difficult problem since the footprint source is often easily changed by external factors. More efficient statistical methods, such as area to area interpolation, might be helpful to solve the problem.

\section{REFERENCES}

Cihlar, J., Chen, J., Li, Z., 1997. On the validation of satellitederived products for land applications. Canadian Journal of Remote Sensing, 23(4), pp. 289-419.

Cochran, W.G., 1977. Sampling Techniques, 3d ed. John Wiley \& Sons, USA.

Ge, Y., Wang, J.H., Wang, J.F., Jin, R., Hu, M.G., 2012. Regression kriging model-based sampling optimization design for the eco-hydrology wireless sensor network. Advances in Earth Science, 27(9), pp. 1006-1013.

Haining, R., 2003. Spatial Data Analysis: Theory and Practice. Cambridge University Press, Cambridge.

Hu, M.G., Wang, J.F., 2011. A spatial sampling optimization package using MSN theory. Environmental Modelling \& Software, 26(4), pp. 546-548.

Jia, Z., S. Liu, Z. Xu, Y. Chen, and M. Zhu, 2012. Validation of remotely sensed evapotranspiration over the HaiRiver Basin, China, Journal of Geophysical Research, 117, D13113.

Jin, R, et al., 2012. Introduction of eco-hydrological wireless sensor network in the Heihe River Basin. Advanced in Earth Science, 27(5), pp. 993-1005.

Justice, C., Belward, A., Morisette, J., Lewis, P., Privette, J., Baret F., 2000. Developments in the 'validation' of satellite sensor products for the study of the land surface. International Journal of Remote Sensing, 21(17), pp. 3383-3390.

Kim, A.Y., Berg, J.C., 2000. Fractal aggregation: Scaling of fractal dimension with stability ratio. Langmuir, 16(5), pp. 2101-2104.

LI, X., Ma M. G., Wang, J., Liu, Q., Che, T., Hu,Z. Y., Xiao, Q., Liu, Q. H., Su, P. X., Chu, R. Z., Jin, R., Wang, W. Z., Ran, Y. H., 2008. Simultaneous Remote Sensing and Ground-based Experiment in the Heihe River Basin: Scientific Objectives and 
Experiment Design, Advances in Earth Science, 23(9), pp. 897914.

Li, X., Li, X.W., Li, Z.Y., Ma, M.G., Wang, J., Xiao, Q., Liu, Q., Che, T., Chen, E.X., Yan, G.J., Hu, Z.Y., Zhang, L.X., Chu, R.Z., Su, P.X., Liu, Q.H., Liu, S.M., Wang, J.D., Niu, Z., Chen, Y., Jin, R., Wang, W.Z., Ran, Y.H., Xin, X.Z. and Ren, H.Z., 2009. Watershed allied telemetry experimental research. Journal of Geophysical Research-Atmospheres, 114(D22).

Li, X, Liu, S.M., Ma, M.G., et al., 2012. HiWATER: An integrated remote sensing experiment on hydrological and ecological processes in the Heihe River Basin. Advances in Earth Science, 27(5), pp. 481-498.

Li, X.W., 2006. Review of the project of quantitative remote sensing of major factors for spatia-temporal heterogeneity on the land surface. Advances in Earth Science, 21(8), pp. 771780 .

Liu, S.M., Li, X.W., Shi, S.J., Xu, Z.W., Bai, J., Ding, X.P., Jia, Z.Z., Zhu, M.J., 2010. Measurement, analysis and application of surface energy and water vapor fluxes at large scale. Advances in Earth Science, 25(11), pp. 1113-1127.

Stein, A., Ettema, C., 2003. An overview of spatial sampling procedures and experimental design of spatial studies for ecosystem comparisons. Agriculture, Ecosystems and Environment, 94(1), pp. 31-47.

Sun, L., Wang, X.M., Guo, M.H., Tang, J.W., 2009. MODIS ocean color product validation around the Yellow Sea and East China Sea. Journal of Lake Science, 21(2), pp. 298-306.

Wang, J.F., B.Y. Reis, M.G. Hu, G. Christakos et al., 2011. Area disease estimation based on sentinel hospital records. PLOS ONE, 6(8), e23428.

Wang, J.F., Christakos, G., Hu, M.G., 2009. Modeling spatial means of surfaces with stratified non-homogeneity. IEEE Transactions on Geoscience and Remote Sensing, 47(12), pp. 4167-4174.

Zhang, R.H., 2009. Quantitative Thermal Infrared Remote Sensing Model and Ground Experimental Basis. Science Press, Peking.

Zhang, R.H., Tian, J., Li, Z.L., Su, H.B., Chen, S.H., 2010. Principles and methods for the validation of quantitative remote sensing products. Science China Earth Sciences, 53(5), pp. 741751.

\section{ACKNOWLEDGEMENTS}

This research was supported in part by the National High Technology Research and Development Program of China (Grant No. 2012AA12A305). 